\title{
Electron localization function implementation in the exact muffin-tin orbitals method
}

\author{
H. Levämäkiఠ* \\ Applied Materials Physics, Department of Materials Science and Engineering, \\ Royal Institute of Technology, Stockholm SE-100 44, Sweden \\ L. Vitos $\odot$ \\ Applied Materials Physics, Department of Materials Science and Engineering, \\ Royal Institute of Technology, Stockholm SE-100 44, Sweden; \\ Department of Physics and Astronomy, Division of Materials Theory, Uppsala University, Box 516, SE-751 20 Uppsala, Sweden; \\ and Research Institute for Solid State Physics and Optics, Wigner Research Center for Physics, Budapest H-1525, Hungary
}

(Received 20 February 2020; revised 1 November 2020; accepted 14 December 2020; published 13 January 2021)

We report implementation of the electron localization function (ELF) within the exact muffin-tin orbitals (EMTO) formalism. The ELF is often used to study the nature of electronic bonding in different types of materials, and it is also an important ingredient in meta-generalized gradient approximations, which are one of the classes of exchange-correlation functionals. The correctness of the ELF implementation is verified with test calculations and comparison with previous literature results. The implementation supports not only regular ordered systems but also disordered systems that have been calculated using the coherent potential approximation method.

\section{DOI: 10.1103/PhysRevB.103.035118}

\section{INTRODUCTION}

The electron localization function (ELF) was put forth by Becke and Edgecombe [1], and it is used to understand the nature of chemical bonding in molecules and periodic systems [2-5]. Studying the ELF can reveal whether the bond between two atoms is covalent, metallic, or ionic and how many electrons each atom contributes to each bond. In chemistry the ELF is often used to understand the topological features of molecules [6]. In solid-state physics, it was recently used to study the effect of local atomic environment on bonding in high-entropy alloys $[7,8]$. In the density functional theory (DFT) $[9,10]$ regime, ELF is also used to describe exchange-correlation (XC) effects, where it serves as one of the inputs to meta-generalized gradient approximations (meta-GGAs) [11-13]. Meta-GGAs are gaining in popularity compared to the standard local density approximations (LDAs) $[10,14-16]$ and generalized gradient approximations (GGAs) [17-23]. The idea behind meta-GGAs is to incorporate the ELF information so that the resulting XC functional can distinguish different parts of space based on the bonding type. With this added flexibility, meta-GGAs are presumably more accurate than LDA and GGA XC functionals, which are not ELF aware.

Based on its usefulness, ELF is a welcome addition to the capabilities of any DFT code. In this paper, we present

\footnotetext{
*levamaki@kth.se
}

Published by the American Physical Society under the terms of the Creative Commons Attribution 4.0 International license. Further distribution of this work must maintain attribution to the author(s) and the published article's title, journal citation, and DOI. Funded by Bibsam. an implementation of the ELF within the exact muffin-tin orbitals (EMTO) DFT method. We derive equations that are needed in EMTO formalism to compute the ELF and after that present the results of a set of test calculations. These results are compared with previously published literature data in order to verify our implementation.

\section{THEORETICAL FORMULATION}

Here and in the following, all physical quantities are expressed in atomic Rydberg units. The ELF distribution is defined as

$$
\eta(\mathbf{r})=\frac{1}{1+\alpha^{2}(\mathbf{r})},
$$

where the $\alpha$ parameter is

$$
\alpha(\mathbf{r})=\frac{\tau(\mathbf{r})-\tau_{\mathrm{W}}(\mathbf{r})}{\tau_{\text {unif }}(\mathbf{r})}
$$

and

$$
\tau_{\text {unif }}(\mathbf{r})=\frac{3}{10}\left(3 \pi^{2}\right)^{2 / 3} n(\mathbf{r})^{5 / 3} .
$$

This definition confines the ELF to always be within the range $0 \leqslant \eta(\mathbf{r}) \leqslant 1$ in such a way that $\eta$ values close to 1 indicate a high degree of electron localization, and the $\eta(\mathbf{r})=1 / 2$ regime corresponds to homogeneous electron gas like Pauli repulsion [24]. $\eta(\mathbf{r})$ values close to zero are not completely clear and should be carefully interpreted [24]. Regions of strong covalent bonding are associated with large ELF values, regions of metallic bonding are associated with $\eta(\mathbf{r}) \approx 1 / 2$, and regions of weak bonding have $\eta(\mathbf{r})<1 / 2$.

Equation (2) shows that ELF depends on three different kinetic energy densities, which are $\tau(\mathbf{r}), \tau_{\mathrm{W}}(\mathbf{r})$, and $\tau_{\text {unif }}(\mathbf{r})$. $\tau(\mathbf{r})$ is the full kinetic energy density (KED) of the electrons, 
and $\tau_{\mathrm{W}}(\mathbf{r})$ is the Weizsäcker KED, which serves as the lower bound for $\tau(\mathbf{r})$, so that $\alpha(\mathbf{r}) \geqslant 0$ always. In a one-electron system $\tau(\mathbf{r})$ and $\tau_{W}(\mathbf{r})$ cancel each other, so that $\alpha(\mathbf{r})=0$. $\tau_{\text {unif }}(\mathbf{r})$ is the KED of the uniform electron gas and is used to normalize $\alpha(\mathbf{r})$ and to make it a dimensionless quantity. The $\alpha$ parameter was also used as a bonding information descriptor in recent meta-GGA functionals, such as strongly constrained and appropriately normed (SCAN), [25] and TaoMo (TM) [26].

Since EMTO is a DFT-based method, here we outline some of the basics of DFT before we get to the EMTO implementation of ELF. DFT uses as the basic variable the electron density

$$
n(\mathbf{r})=\sum_{j, \epsilon_{j} \leqslant \epsilon_{F}} \Psi_{j}^{*}(\mathbf{r}) \Psi_{j}(\mathbf{r}),
$$

which is calculated as a sum of the occupied Kohn-Sham (KS) orbitals $\Psi_{j}$ up to the Fermi level $\epsilon_{F}$. For simplicity, we leave out spin indexing from our equations and assume that each KS orbital is fully occupied up to the Fermi level. KS orbitals are solutions to the KS equations

$$
\left\{-\frac{1}{2} \nabla^{2}+V_{\mathrm{KS}}(\mathbf{r})\right\} \Psi_{j}(\mathbf{r})=\epsilon_{j} \Psi_{j}(\mathbf{r})
$$

for some specific KS orbital energies $\epsilon_{j} . V_{\mathrm{KS}}(\mathbf{r})$ is the $\mathrm{KS}$ effective potential. The total energy is a functional of the density and is written as

$$
E[n]=T_{s}[n]+\int V_{\text {ext }}(\mathbf{r}) n(\mathbf{r}) d \mathbf{r}+E_{\text {Hartree }}[n]+E_{\mathrm{xc}}[n]+E_{I I},
$$

where $T_{s}[n]$ is the noninteracting kinetic energy, $V_{\text {ext }}$ is the external potential, $E_{\text {Hartree }}$ is the Coulombic interaction of the density with itself, $E_{\mathrm{xc}}$ is the XC interaction energy, and $E_{I I}$ is the interaction energy between the static (Born-Oppenheimer approximation) atomic nuclei. The $\operatorname{KED} \tau(\mathbf{r})$ is defined as

$$
T_{s}=\int \tau(\mathbf{r}) d \mathbf{r}
$$

It is important to note that the definition of $\tau(\mathbf{r})$ in Eq. (7) is not unique because we can add to $\tau(\mathbf{r})$ any function that integrates to zero. There are two important forms for KED [27]. The first depends on the Laplacian of the KS orbitals $\Psi_{j}(\mathbf{r})$ :

$$
\tau^{L}(\mathbf{r})=-\frac{1}{2} \sum_{j, \epsilon_{j} \leqslant \epsilon_{F}} \Psi_{j}(\mathbf{r})^{*} \nabla^{2} \Psi_{j}(\mathbf{r}),
$$

and it is derived directly from the KS equations. The second one is positive definite:

$$
\begin{aligned}
\tau(\mathbf{r}) & =\frac{1}{2} \sum_{j, \epsilon_{j} \leqslant \epsilon_{F}} \nabla \Psi_{j}^{*}(\mathbf{r}) \cdot \nabla \Psi_{j}(\mathbf{r}) \\
& =\frac{1}{2} \sum_{j, \epsilon_{j} \leqslant \epsilon_{F}}\left|\nabla \Psi_{j}(\mathbf{r})\right|^{2}=\tau^{L}(\mathbf{r})+\frac{1}{4} \nabla^{2} n(\mathbf{r}) .
\end{aligned}
$$

Defining $\tau(\mathbf{r})$ using Eq. (9) is the popular choice because then $\tau(\mathbf{r})$ is guaranteed to be positive everywhere, and for a singleorbital system this definition leads to the so-called Weizsäcker term,

$$
\tau_{W}(\mathbf{r})=\frac{1}{8} \frac{\nabla n(\mathbf{r}) \cdot \nabla n(\mathbf{r})}{n(\mathbf{r})}
$$

Due to these desirable features we will define $\tau(\mathbf{r})$ using Eq. (9) in our EMTO implementation.

\section{A. EMTO formalism}

The EMTO is part of the large family of muffin-tin orbital methods. The muffin-tin approximation divides the space into atom-centered spheres, called muffin-tins, and space outside the spheres, called the interstitial region. Such a division makes the problem of solving KS equations more manageable because it is easier to solve the KS equations separately inside the muffin-tin spheres and in the interstitial region and then stitch the two solutions together at the boundaries. The EMTO formalism is part of the third generation of muffin-tin orbitals methods [28]. The first generation was the linear muffin-tin orbitals (LMTO) method [29,30], which introduced a highly efficient, minimal, energy-independent muffin-tin orbitals basis set. The second generation introduced screening for the muffin-tin orbitals, which led to the computationally fast tightbinding LMTO formalism [31]. The third generation fixes the weak point of the earlier generations and solves the interstitial region with a level of accuracy similar to that with which the insides of the muffin-tin spheres are solved [28]. For more information about the specifics of the EMTO formalism the reader is referred to, e.g., Refs. [28,32-35].

In EMTO the KS orbitals are expanded in the basis of EMTOs $\bar{\psi}_{R L}^{a}\left(\epsilon_{j}, \mathbf{r}_{R}\right)$, so we write

$$
\Psi_{j}(\mathbf{r})=\sum_{R L} \bar{\psi}_{R L}^{a}\left(\epsilon_{j}, \mathbf{r}_{R}\right) v_{R L, j}^{a},
$$

where $v_{R L, j}^{a}$ are the expansion coefficients and the notation $\mathbf{r}_{R}=\mathbf{r}-\mathbf{R}$ defines coordinates relative to each lattice site $R$. The EMTOs $\bar{\psi}_{R L}^{a}\left(\epsilon_{j}, \mathbf{r}_{R}\right)$ are defined differently inside and outside of the muffin-tin spheres. The total EMTO is then constructed by fitting the different definitions together in such a way that the EMTO is continuous everywhere, but the derivative has a kink at the so-called hard-sphere boundaries. Since a KS orbital is a sum of EMTOs and must be continuous and differentiable everywhere, the coefficients $v_{R L, j}^{a}$ in the sum of EMTOs must be chosen in such a way that the sum of EMTO kinks vanishes. Requiring the kink of the KS orbital $\Psi_{j}(\mathbf{r})$ to vanish leads to the kink-cancellation matrix equation

$$
\mathbf{K}^{a}\left(\epsilon_{j}\right) \cdot \mathbf{v}_{j}^{a}=\mathbf{0},
$$

where $\mathbf{v}_{j}^{a}$ is a column vector of the expansion coefficients $v_{R L, j}^{a}$ and $\mathbf{K}^{a}\left(\epsilon_{j}\right)$ is known as the kink matrix. The elements of the kink matrix describe the size of the derivative kink of each EMTO.

In the current implementation of EMTO we use a Green's function [36] to solve the KS equations and to calculate physical observables. Even though the Green's function formalism is computationally demanding for ordered systems, the benefit is that it provides, together with the coherent potential approximation (CPA) [37-39], a highly elegant framework to treat disordered systems. The Green's function will be constructed using the so-called path operator $\mathbf{g}^{a}(z)$, which is defined to be the inverse of the kink matrix,

$$
\mathbf{K}^{a}(z) \mathbf{g}^{a}(z)=\mathbf{I} .
$$


It can be shown that $\mathbf{g}^{a}(z)$ has poles only at the KS eigenenergies $z=\epsilon_{j}$, which allows us to construct the Green's function using the path operator.

A properly normalized first-order density matrix within one Wigner-Seitz (WS) cell is, in EMTO, written as

$$
\begin{aligned}
n\left(\mathbf{r}_{R}, \mathbf{r}_{R}^{\prime}\right) & =\sum_{j, \epsilon_{j} \leqslant \epsilon_{F}} \frac{\Psi_{j}^{*}\left(\epsilon_{j}, \mathbf{r}_{R}^{\prime}\right) \Psi_{j}\left(\epsilon_{j}, \mathbf{r}_{R}\right)}{\int \Psi_{j}^{*}\left(\epsilon_{j}, \mathbf{r}_{R}\right) \Psi_{j}\left(\epsilon_{j}, \mathbf{r}_{R}\right) d \mathbf{r}} \\
& =\sum_{j, \epsilon_{j} \leqslant \epsilon_{F}} \frac{\Psi_{j}^{*}\left(\epsilon_{j}, \mathbf{r}_{R}^{\prime}\right) \Psi_{j}\left(\epsilon_{j}, \mathbf{r}_{R}\right)}{\sum_{R^{\prime} L^{\prime} R L} v_{R^{\prime} L^{\prime}, j}^{a *} \dot{K}_{R^{\prime} L^{\prime} R L}\left(\epsilon_{j}\right) v_{R L, j}^{a}},
\end{aligned}
$$

where the matrix $\dot{\mathbf{K}}^{a}(z)$ is the energy derivative of the kink matrix but also gives the overlap between two EMTOs as $\dot{K}_{R^{\prime} L^{\prime} R L}^{a}(z)=\int \bar{\psi}_{R^{\prime} L^{\prime}}^{*}(z, \mathbf{r}) \bar{\psi}_{R L}(z, \mathbf{r}) d \mathbf{r}$. With the help of the path operator and the residue theorem, the density matrix can be calculated as

$$
\begin{aligned}
n\left(\mathbf{r}_{R}, \mathbf{r}_{R}^{\prime}\right)= & \frac{1}{2 \pi i} \oint_{\epsilon_{F}} \sum_{L^{\prime \prime} L^{\prime}} Z_{R l^{\prime}}^{a}\left(z, r_{R}^{\prime}\right) \tilde{g}_{R L^{\prime \prime} L^{\prime}}^{a}(z) \\
& \times Z_{R l^{\prime \prime}}^{a}\left(z, r_{R}\right) Y_{L^{\prime}}\left(\hat{\mathbf{r}}_{R}^{\prime}\right) Y_{L^{\prime \prime}}\left(\hat{\mathbf{r}}_{R}\right) d z
\end{aligned}
$$

where the $Z_{R l}^{a}\left(z, r_{R}\right)$ functions are defined in terms of the EMTOs $\bar{\psi}_{R L}^{a}\left(z, \mathbf{r}_{R}\right), Y_{L}\left(\hat{\mathbf{r}}_{R}\right)$ are the real harmonics, and $\tilde{g}_{R L^{\prime \prime} L^{\prime}}^{a}(z)$ is defined in terms of the path operator $g_{R^{\prime} L^{\prime} R L}^{a}(z)$ and the so-called slope matrix $S_{R^{\prime} L^{\prime} R L}^{a}(z)$, which is related to the well-known Korringa-Kohn-Rostoker structure constant matrix [34]. $\tilde{g}_{R L^{\prime \prime} L^{\prime}}^{a}(z)$ also ensures that the so-called unphysical poles are removed, so that only the poles caused by the physically meaningful $z=\epsilon_{j}$ KS solutions are processed by the residue theorem. These unphysical poles are caused by a normalization function in the $Z_{R l}^{a}\left(z, r_{R}\right)$ term, and the scheme for their removal can be found in Ref. [34]. Equation (15) yields the electron density as $n\left(\mathbf{r}_{R}\right)=n\left(\mathbf{r}_{R}, \mathbf{r}_{R}\right)$.

The KED matrix $\tau\left(\mathbf{r}_{R}, \mathbf{r}_{R}^{\prime}\right)$ can now be obtained from the density matrix by applying a gradient operator $\frac{1}{2} \nabla_{\mathbf{r}_{R}} \nabla_{\mathbf{r}_{R}^{\prime}}$. Hence, we can write

$$
\begin{aligned}
\tau\left(\mathbf{r}_{R}, \mathbf{r}_{R}^{\prime}\right) & =\frac{1}{2} \nabla_{\mathbf{r}_{R}} \nabla_{\mathbf{r}_{R}^{\prime}} n\left(\mathbf{r}_{R}, \mathbf{r}_{R}^{\prime}\right) \\
& =\frac{1}{2} \sum_{j, \epsilon_{j} \leqslant \epsilon_{F}} \frac{\nabla_{\mathbf{r}_{R}^{\prime}} \Psi_{j}^{*}\left(\epsilon_{j}, \mathbf{r}_{R}^{\prime}\right) \cdot \nabla_{\mathbf{r}_{R}} \Psi_{j}\left(\epsilon_{j}, \mathbf{r}_{R}\right)}{\sum_{R^{\prime} L^{\prime} R L} v_{R^{\prime} L^{\prime}, j}^{a *} \dot{K}_{R^{\prime} L^{\prime} R L}\left(\epsilon_{j}\right) v_{R L, j}^{a}} .
\end{aligned}
$$

Using Eq. (15), we get

$$
\begin{aligned}
\tau\left(\mathbf{r}_{R}, \mathbf{r}_{R}^{\prime}\right)= & \frac{1}{4 \pi i} \oint_{\epsilon_{F}} \sum_{L^{\prime \prime} L^{\prime}} \tilde{g}_{R L^{\prime \prime} L^{\prime}}^{a}(z) \nabla_{\mathbf{r}_{R}^{\prime}}\left[Z_{R l^{\prime}}^{a}\left(z, r_{R}^{\prime}\right) Y_{L^{\prime}}\left(\hat{\mathbf{r}}_{R}^{\prime}\right)\right] \\
& \cdot \nabla_{\mathbf{r}_{R}}\left[Z_{R l^{\prime \prime}}^{a}\left(z, r_{R}\right) Y_{L^{\prime \prime}}\left(\hat{\mathbf{r}}_{R}\right)\right] d z .
\end{aligned}
$$

From Eq. (17) we obtain the KED within the WS cell by setting $\mathbf{r}_{R}^{\prime}=\mathbf{r}_{R}$ :

$$
\begin{aligned}
\tau\left(\mathbf{r}_{R}\right)= & \frac{1}{4 \pi i} \oint_{\epsilon_{F}} \sum_{L^{\prime \prime} L^{\prime}} \tilde{g}_{R L^{\prime \prime} L^{\prime}}^{a}(z) \nabla\left[Z_{R l^{\prime}}^{a}\left(z, r_{R}\right) Y_{L^{\prime}}\left(\hat{\mathbf{r}}_{R}\right)\right] \\
& \cdot \nabla\left[Z_{R l^{\prime \prime}}^{a}\left(z, r_{R}\right) Y_{L^{\prime \prime}}\left(\hat{\mathbf{r}}_{R}\right)\right] d z .
\end{aligned}
$$

The gradient of $Z_{R l}^{a}\left(z, r_{R}\right) Y_{L}\left(\hat{\mathbf{r}}_{R}\right)$ is calculated in spherical coordinates, and we can therefore write

$$
\begin{aligned}
& \nabla\left[Z_{R l^{\prime}}^{a}\left(z, r_{R}\right) Y_{L^{\prime}}\left(\hat{\mathbf{r}}_{R}\right)\right] \\
& =\frac{\partial Z_{R l^{\prime}}^{a}\left(z, r_{R}\right)}{\partial r_{R}} Y_{L^{\prime}}\left(\hat{\mathbf{r}}_{R}\right) \mathbf{e}_{r}+\frac{Z_{R l^{\prime}}^{a}\left(z, r_{R}\right)}{r_{R}} \frac{\partial Y_{L^{\prime}}\left(\hat{\mathbf{r}}_{R}\right)}{\partial \theta_{R}} \mathbf{e}_{\theta} \\
& \quad+\frac{Z_{R l^{\prime}}^{a}\left(z, r_{R}\right)}{r_{R} \sin \theta_{R}} \frac{\partial Y_{L^{\prime}}\left(\hat{\mathbf{r}}_{R}\right)}{\partial \phi_{R}} \mathbf{e}_{\phi} .
\end{aligned}
$$

The dot product between the two gradients in Eq. (18) is straightforwardly evaluated using Eq. (19).

Equation (18) gives the KED inside one WS cell as a double- $L$ sum, which can make evaluating the KED and ELF in real space, e.g., for plotting purposes, quite slow. For practical reasons we want to have the KED in one-center form, which is to say, we expand $\tau\left(\mathbf{r}_{R}\right)$ in terms of real harmonics as

$$
\tau_{R}\left(\mathbf{r}_{R}\right)=\sum_{L} \tau_{R L}\left(r_{R}\right) Y_{L}\left(\hat{\mathbf{r}}_{R}\right)
$$

The one-center form $\tau_{R}\left(\mathbf{r}_{R}\right)$ contains only a single- $L$ sum, which makes evaluating the KED in real space much faster. Since the WS cells cover the whole space without any overlap, the total KED is formally given by the sum of one-center expansions as

$$
\tau(\mathbf{r})=\sum_{R} \tau_{R}\left(\mathbf{r}_{R}\right)
$$

The one-center expansion coefficients are calculated by projecting $\tau\left(\mathbf{r}_{R}\right)$ into each real harmonic $Y_{L}$, so the formula to calculate the expansion coefficient is

$$
\tau_{R L}\left(r_{R}\right)=\int \tau\left(\mathbf{r}_{R}\right) Y_{L}\left(\hat{\mathbf{r}}_{R}\right) d \Omega
$$

where $d \Omega$ means integrating over the $\theta$ and $\phi$ angles of the spherical coordinates. By inserting Eqs. (18) and (19) into Eq. (22) we get

$$
\begin{aligned}
\tau_{R L}\left(r_{R}\right)= & \frac{1}{4 \pi i} \oint_{\epsilon_{F}} \sum_{L^{\prime \prime} L^{\prime}} C_{L L^{\prime} L^{\prime \prime}} \tilde{g}_{R L^{\prime \prime} L^{\prime}}^{a}(z) \frac{\partial Z_{R l^{\prime}}^{a}\left(z, r_{R}\right)}{\partial r_{R}} \frac{\partial Z_{R l^{\prime \prime}}^{a}\left(z, r_{R}\right)}{\partial r_{R}} d z \\
& +\frac{1}{4 \pi i} \oint_{\epsilon_{F}} \sum_{L^{\prime \prime} L^{\prime}} \tilde{g}_{R L^{\prime \prime} L^{\prime}}^{a}(z) \frac{Z_{R l^{\prime}}^{a}\left(z, r_{R}\right)}{r_{R}} \frac{Z_{R l^{\prime \prime}}^{a}\left(z, r_{R}\right)}{r_{R}} \int \frac{\partial Y_{L^{\prime}}\left(\hat{\mathbf{r}}_{R}\right)}{\partial \theta_{R}} \frac{\partial Y_{L^{\prime \prime}}\left(\hat{\mathbf{r}}_{R}\right)}{\partial \theta_{R}} Y_{L}\left(\hat{\mathbf{r}}_{R}\right) d \Omega d z \\
& +\frac{1}{4 \pi i} \oint_{\epsilon_{F}} \sum_{L^{\prime \prime} L^{\prime}} \tilde{g}_{R L^{\prime \prime} L^{\prime}}^{a}(z) \frac{Z_{R l^{\prime}}^{a}\left(z, r_{R}\right)}{r_{R}} \frac{Z_{R l^{\prime \prime}}^{a}\left(z, r_{R}\right)}{r_{R}} \int \frac{1}{\sin ^{2} \theta_{R}} \frac{\partial Y_{L^{\prime}}\left(\hat{\mathbf{r}}_{R}\right)}{\partial \phi_{R}} \frac{\partial Y_{L^{\prime \prime}}\left(\hat{\mathbf{r}}_{R}\right)}{\partial \phi_{R}} Y_{L}\left(\hat{\mathbf{r}}_{R}\right) d \Omega d z,
\end{aligned}
$$


where $C_{L L^{\prime} L^{\prime \prime}}$ are the real Gaunt numbers, which arise from the integral of three real harmonics as

$$
C_{L L^{\prime} L^{\prime \prime}}=\int Y_{L}\left(\hat{\mathbf{r}}_{R}\right) Y_{L^{\prime}}\left(\hat{\mathbf{r}}_{R}\right) Y_{L^{\prime \prime}}\left(\hat{\mathbf{r}}_{R}\right) d \Omega
$$

In principle the angular integrals in Eqs. (24) and (25) can be solved analytically using the definition of real harmonics and the recursion formulas of the Legendre polynomials, but doing so would be rather tedious. Instead, we choose to solve these angle integrals numerically, which is straightforward.

In addition to $\tau(\mathbf{r})$ we also need $\tau_{\text {unif }}(\mathbf{r})$ and $\tau_{\mathrm{W}}(\mathbf{r})$ to get the ELF. $\tau_{\text {unif }}(\mathbf{r})$ is easily calculated using Eq. (3) and the onecenter expansion of the density, which is already implemented in the EMTO code. In principle, the Weizsäcker term $\tau_{W}\left(\mathbf{r}_{R}\right)=$ $1 / 8\left[\nabla n\left(\mathbf{r}_{R}\right) \cdot \nabla n\left(\mathbf{r}_{R}\right)\right] / n\left(\mathbf{r}_{R}\right)$ could also be computed directly from the one-center expansion of $n(\mathbf{r})$ by applying the gradient operator $\nabla$ defined in spherical coordinates. However, we have found that the direct evaluation of $\tau_{W}(\mathbf{r})$ from the one-center expansion of $n(\mathbf{r})$ can cause inconsistency in the $\tau(\mathbf{r})-\tau_{\mathrm{W}}(\mathbf{r})$ numerator expression that $\alpha(\mathbf{r})$ is calculated from in Eq. (2). Maximum consistency is obtained by calculating $\tau_{W}\left(\mathbf{r}_{R}\right)$ the same way that the one-center expansion of $\tau(\mathbf{r})$ is calculated, in the spirit of Eqs. (23)-(25). To this end, the gradient of $n\left(\mathbf{r}_{R}\right)$ can be written as

$$
\begin{aligned}
\nabla n\left(\mathbf{r}_{R}\right)= & \frac{1}{2 \pi i} \oint_{\epsilon_{F}} \sum_{L^{\prime \prime} L^{\prime}}\left[\frac{\partial Z_{R l^{\prime}}^{a}\left(z, r_{R}\right)}{\partial r_{R}} Z_{R l^{\prime \prime}}^{a}\left(z, r_{R}\right)+Z_{R l^{\prime}}^{a}\left(z, r_{R}\right) \frac{\partial Z_{R l^{\prime \prime}}^{a}\left(z, r_{R}\right)}{\partial r_{R}}\right] \tilde{g}_{R L^{\prime \prime} L^{\prime}}^{a}(z) Y_{L^{\prime}}\left(\hat{\mathbf{r}}_{R}\right) Y_{L^{\prime \prime}}\left(\hat{\mathbf{r}}_{R}\right) d z \mathbf{e}_{r} \\
& +\frac{1}{2 \pi i} \oint_{\epsilon_{F}} \sum_{L^{\prime \prime} L^{\prime}} \frac{Z_{R l^{\prime}}^{a}\left(z, r_{R}\right) Z_{R l^{\prime \prime}}^{a}\left(z, r_{R}\right)}{r_{R}} \tilde{g}_{R L^{\prime \prime} L^{\prime}}^{a}(z)\left[\frac{\partial Y_{L^{\prime}}\left(\hat{\mathbf{r}}_{R}\right)}{\partial \theta_{R}} Y_{L^{\prime \prime}}\left(\hat{\mathbf{r}}_{R}\right)+Y_{L^{\prime}}\left(\hat{\mathbf{r}}_{R}\right) \frac{\partial Y_{L^{\prime \prime}}\left(\hat{\mathbf{r}}_{R}\right)}{\partial \theta_{R}}\right] d z \mathbf{e}_{\theta} \\
& +\frac{1}{2 \pi i} \oint_{\epsilon_{F}} \sum_{L^{\prime \prime} L^{\prime}} \frac{Z_{R l^{\prime}}^{a}\left(z, r_{R}\right) Z_{R l^{\prime \prime}}^{a}\left(z, r_{R}\right)}{r_{R} \sin \theta_{R}} \tilde{g}_{R L^{\prime \prime} L^{\prime}}^{a}(z)\left[\frac{\partial Y_{L^{\prime}}\left(\hat{\mathbf{r}}_{R}\right)}{\partial \phi_{R}} Y_{L^{\prime \prime}}\left(\hat{\mathbf{r}}_{R}\right)+Y_{L^{\prime}}\left(\hat{\mathbf{r}}_{R}\right) \frac{\partial Y_{L^{\prime \prime}}\left(\hat{\mathbf{r}}_{R}\right)}{\partial \phi_{R}}\right] d z \mathbf{e}_{\phi} .
\end{aligned}
$$

$\nabla n\left(\mathbf{r}_{R}\right)$ is symmetric with respect to the $L^{\prime \prime}$ and $L^{\prime}$ indices, which means we can save some computation time by calculating only the $L^{\prime \prime}>L^{\prime}$ terms and multiplying them by 2 . The one-center coefficients of the components of the $\nabla n\left(\mathbf{r}_{R}\right)$ vector become

$$
\begin{gathered}
\nabla n_{R L}^{\mathbf{e}_{r}}\left(r_{R}\right)=\frac{1}{2 \pi i} \oint_{\epsilon_{F}} \sum_{L^{\prime \prime} L^{\prime}} C_{L L^{\prime} L^{\prime \prime}}\left[\frac{\partial Z_{R l^{\prime}}^{a}\left(z, r_{R}\right)}{\partial r_{R}} Z_{R l^{\prime \prime}}^{a}\left(z, r_{R}\right)+Z_{R l^{\prime}}^{a}\left(z, r_{R}\right) \frac{\partial Z_{R l^{\prime \prime}}^{a}\left(z, r_{R}\right)}{\partial r_{R}}\right] \tilde{g}_{R L^{\prime \prime} L^{\prime}}^{a}(z) d z, \\
\nabla n_{R L}^{\mathbf{e}_{\theta}}\left(r_{R}\right)=\frac{1}{2 \pi i} \oint_{\epsilon_{F}} \sum_{L^{\prime \prime} L^{\prime}} \frac{Z_{R l^{\prime}}^{a}\left(z, r_{R}\right) Z_{R l^{\prime \prime}}^{a}\left(z, r_{R}\right)}{r_{R}} \tilde{g}_{R L^{\prime \prime} L^{\prime}}^{a}(z) \int Y_{L}\left(\hat{\mathbf{r}}_{R}\right)\left[\frac{\partial Y_{L^{\prime}}\left(\hat{\mathbf{r}}_{R}\right)}{\partial \theta_{R}} Y_{L^{\prime \prime}}\left(\hat{\mathbf{r}}_{R}\right)+Y_{L^{\prime}}\left(\hat{\mathbf{r}}_{R}\right) \frac{\partial Y_{L^{\prime \prime}}\left(\hat{\mathbf{r}}_{R}\right)}{\partial \theta_{R}}\right] d \Omega d z, \\
\nabla n_{R L}^{\mathbf{e}_{\phi}}\left(r_{R}\right)=\frac{1}{2 \pi i} \oint_{\epsilon_{F}} \sum_{L^{\prime \prime} L^{\prime}} \frac{Z_{R l^{\prime}}^{a}\left(z, r_{R}\right) Z_{R l^{\prime \prime}}^{a}\left(z, r_{R}\right)}{r_{R} \sin \theta_{R}} \tilde{g}_{R L^{\prime \prime} L^{\prime}}^{a}(z) \int Y_{L}\left(\hat{\mathbf{r}}_{R}\right)\left[\frac{\partial Y_{L^{\prime}}\left(\hat{\mathbf{r}}_{R}\right)}{\partial \phi_{R}} Y_{L^{\prime \prime}}\left(\hat{\mathbf{r}}_{R}\right)+Y_{L^{\prime}}\left(\hat{\mathbf{r}}_{R}\right) \frac{\partial Y_{L^{\prime \prime}}\left(\hat{\mathbf{r}}_{R}\right)}{\partial \phi_{R}}\right] d \Omega d z .
\end{gathered}
$$

Only the radial $\nabla n_{R L}^{\mathbf{e}_{r}}\left(r_{R}\right)$ component allows simplification using Gaunt numbers. The angular integrals of the $\nabla n_{R L}^{\mathbf{e}_{\theta}}\left(r_{R}\right)$ and $\nabla n_{R L}^{\mathbf{e}_{\phi}}\left(r_{R}\right)$ components are computed numerically. One can go further and compute the one-center expansions for $\tau_{W}\left(\mathbf{r}_{R}\right)$ and $\eta\left(\mathbf{r}_{R}\right)$, but they are also quite easily evaluated at any given point in space by using the one-center expansions of $n\left(\mathbf{r}_{R}\right)$, $\nabla n\left(\mathbf{r}_{R}\right)$, and $\tau\left(\mathbf{r}_{R}\right)$.

The EMTO code allows one to simulate a disordered system by using the CPA, which replaces the real disordered system by an ordered effective medium [34]. It is therefore possible to calculate the ELF of disordered systems using the CPA. Since the symmetry of the underlying crystal structure is kept, the power of the CPA is that it allows one to simulate disorder using minimal unit cell sizes. Within the CPA, the total energy is a concentration weighted average of the CPA component energies. The CPA electron density is defined for each CPA component separately, and the one-center density of component $i$ is written as

$$
n_{R}^{i}\left(\mathbf{r}_{R}\right)=\sum_{L} n_{R L}^{i}\left(r_{R}\right) Y_{L}\left(\hat{\mathbf{r}}_{R}\right)
$$

The extension of ELF for CPA is thus straightforward. We simply use the equations presented above and compute $\tau_{R}^{i}\left(\mathbf{r}_{R}\right)$, $\tau_{W, R}^{i}\left(\mathbf{r}_{R}\right), \tau_{\text {unif }, R}^{i}\left(\mathbf{r}_{R}\right)$, and $\eta_{R}^{i}\left(\mathbf{r}_{R}\right)$ for each CPA component. The physical significance of CPA ELF will be discussed in a future work.

\section{B. Technical implementation details}

Due to the approximations made in EMTO, specifically, the $l$ truncations in one-center expansions, the ELF may appear discontinuous at the WS (Voronoi) cell boundaries between atoms. Since EMTO uses overlapping potential spheres, the ELF within the overlap regions that contain the WS boundaries is not, in a sense, uniquely defined. To get a "uniquely" defined ELF we employ an averaging technique, which is based on the fuzzy cells concept of Becke [40]. We present the ELF as

$$
\eta(\mathbf{r})=\sum_{R} w_{R}(\mathbf{r}) \eta\left(\mathbf{r}_{R}\right)
$$

where each lattice site $R$ has an appropriately chosen weight function $w_{R}(\mathbf{r})$. These weight functions should satisfy the condition $\sum_{R} w_{R}(\mathbf{r})=1$, so we define the weights to be

$$
w_{R}(\mathbf{r})=\frac{P_{R}(\mathbf{r})}{\sum_{R^{\prime}} P_{R^{\prime}}(\mathbf{r})},
$$

where $P_{R}(\mathbf{r})$ are lattice site centered partial weights. The boundary conditions that $P_{R}(\mathbf{r})$ must obey are the require- 
ments that at the lattice site $R P_{R}(\mathbf{r})=1$ and far away from the lattice site $P_{R}(\mathbf{r})$ decays to zero. The partial weights have been defined many different ways in the literature, and here we define them following one of our previous publications [41] and write

$$
P_{R}(\mathbf{r})=\exp \left[-\left(\frac{|\mathbf{r}-\mathbf{R}|}{\lambda}\right)^{2 \beta}\right]
$$

where $\beta$ controls how sharply each $P_{R}(\mathbf{r})$ decays from 1 to 0 and $\lambda$ controls the distance from the lattice site $R$ where the decay happens. Small $\beta$ values make the partial weights longer ranged, which increases the amount of averaging. When $\beta$ is large ( $\gtrsim 1.8$ ), the weight fields $w_{R}(\mathbf{r})$ divide the space sharply into Voronoi cells, and there will be no averaging. The value of $\lambda$ should be chosen in such a way that $P_{R}(\mathbf{r})$ functions have strong enough exponential decay close to the Voronoi cell boundaries. Here we use the same parameter values as in our earlier work [41] ( $\lambda=1.2 \mathrm{bohr}$ and $\beta=2.0)$ since they fulfill the decay condition for solids close to their equilibrium state.

\section{Numerical details}

All calculations used LDA to describe the exchange and correlation effects. EMTO calculations were performed within the scalar-relativistic and soft-core schemes. The EMTO basis set included the $s, p, d$, and $f$ orbitals, and the one-center expansions were calculated up to cutoff value $l_{\max }^{h}=28$. The Green's function was calculated for 16 complex energy points distributed exponentially on a semicircular contour. $k$-point meshes were selected in such a way that there were $\approx 1500 k$ points in the irreducible wedge of the Brillouin zone. In the fcc $\mathrm{Cu}$ and fcc CuZn CPA calculations the muffin-tin zero has been shifted up by $0.2 \mathrm{Ry}$.

We also carried out reference calculations using the full-potential linearized augmented plane wave method as implemented in the ELK code [42]. The ELK calculations were performed using conventional representations of the fcc and $B 2$ structures with $10 \times 10 \times 10 k$-point meshes. Recommended input settings for obtaining an accurate ELF were used: LRADSTP $=1$, RGKMAX $=11$, GMAXVR $=22$, MSMOOTH $=4$, LMAXAPW $=12$, LMAXO $=12$. Default species input parameters were used in all ELK calculations.

\section{About the $l$ convergence of the one-center expansions}

The accuracy of a one-center expansion, such as that for $\tau$ in Eq. (20), is governed by the cutoff value $l_{\max }^{h}$, which defines how many $L=\{l, m\}$ terms are included in the expansion. Because there are $2 l+1$ magnetic quantum numbers $m$ for each value of $l$, the expansion becomes computationally increasingly demanding to calculate when larger and larger $l$ terms are included in the expansion.

On the other hand, while the total energy is described with adequate accuracy using a cutoff $l_{\max }^{h}=8$, we have found that obtaining an accurate ELF everywhere in real space requires a noticeably larger $l$ cutoff. The reason for this has to do with the fact that the one-center expansion becomes less and less accurate as we move away from the nucleus, which is the center of the expansion. Therefore, if a crystal structure is de-
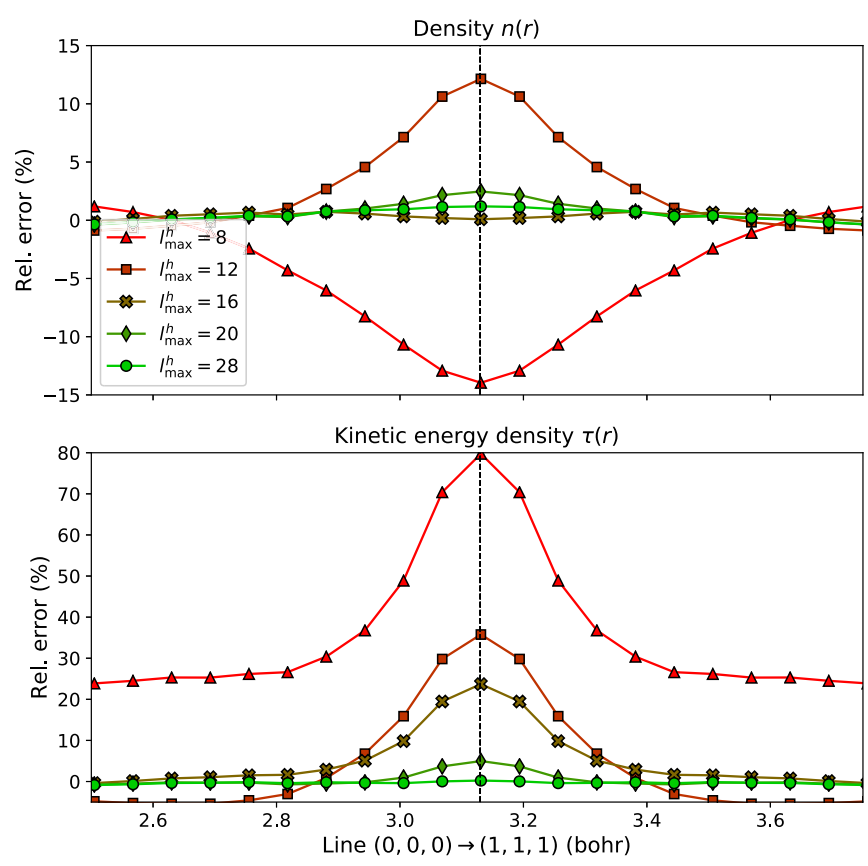

FIG. 1. Convergence of the density and KED one-center expansions for fcc $\mathrm{Cu}$ as a function of the cutoff parameter $l_{\mathrm{max}}^{h}$. The curves represent relative errors between EMTO and elk quantities plotted along a line from the $(0,0,0)$ coordinate to the $(1,1,1)$ coordinate of the conventional fcc unit cell. Only the critical region around the center of the interstitial region (vertical dashed line) at unit cell coordinate $(1 / 2,1 / 2,1 / 2)$ is shown.

scribed by Voronoi cells that have elongated shapes, i.e., some corners of the Voronoi cell are far away from the nucleus, a large $l$ cutoff will be needed to get an accurate ELF near those far-away corners. This is the situation, for example, in the fcc structure, where the corners of the Voronoi cells meet in the center of the interstitial region and the shape of the fcc Voronoi cell is somewhat elongated. In other structures, for instance, bcc, the problem is much smaller because the shape of the bcc Voronoi cell is quite round.

A proper $l$ cutoff for an accurate ELF in the fcc structure is therefore established here. Because the ELF is calculated from the one-center expansions of the density and the KED, we should make sure that both of those one-center expansions are accurately described. Figure 1 depicts the $l$ convergence of the density and KED around the center of the interstitial region in $\mathrm{fcc} \mathrm{Cu}$. We can see that compared to reference quantities produced by the full-potential code ELK, while $l_{\max }^{h}=16$ happens to give an acceptably low relative error with respect to the density $\left(\left[n(\mathbf{r})_{\text {EMTO }}-n(\mathbf{r})_{\text {elk }}\right] / n(\mathbf{r})_{\text {elk }}\right)$, the relative error of the KED has not yet converged. It can be seen that the cutoff should be increased to $l_{\max }^{h}=28$ to achieve good convergence for both the density and the KED. Throughout the paper we will therefore use a cutoff value $l_{\max }^{h}=28$ in our calculations.

\section{TEST CALCULATIONS}

\section{A. Kinetic energy of elemental metals}

As a first test, we calculate the total kinetic energy $T_{s}$ two different ways: the way the EMTO code normally calculates 
TABLE I. Comparison of nonrelativistic kinetic energies calculated two different ways: (1) using the KS equations (EMTO) and (2) integrating the $\operatorname{KED}\left[\int \tau(\mathbf{r}) d \mathbf{r}\right]$. The values reported are per unit cell and in units of rydbergs.

\begin{tabular}{lrr}
\hline \hline & EMTO & $\int \tau(\mathbf{r}) d \mathbf{r}$ \\
\hline bcc Li & 14.6 & 14.6 \\
fcc Al & 481.4 & 481.6 \\
fcc Ca & 1349.3 & 1349.2 \\
fcc Cu & 3272.1 & 3272.7 \\
\hline \hline
\end{tabular}

it, i.e., by making use of the Kohn-Sham single-electron equations, and by numerically integrating the calculated one-center expansion of $\tau(\mathbf{r})$ within the unit cell using the shape function technique [34]. Table I provides the results per unit cell for $\mathrm{Li}$ in a bec structure and $\mathrm{Al}, \mathrm{Ca}$, and $\mathrm{Cu}$ in a fcc structure. Since EMTO kinetic energy normally includes the scalar-relativistic effects, while kinetic energy calculated by numerically integrating $\tau(\mathbf{r})$ does not, EMTO calculations for this test have been run nonrelativistically to make the kinetic energies comparable. The numerical integral used 100 grid points for the angular $\theta$ and $\phi$ dimensions and 3000 grid points to describe the radial part of the integral. We can see that the two kinetic energies agree well within some error bars. The reason for small differences is that while the EMTO kinetic energy is computed as the difference between the one electron energies (computed via integration over the Brillouin zone) and the potential term, the direct calculation is performed using the shape function technique. In addition, the EMTO kinetic energy suffers from the overlap error (one electron states are normalized using a nonoverlapping overlap matrix), while the direct calculation contains no explicit overlap contribution.

\section{B. ELF for monoatomic and diatomic systems}

To test the correctness and accuracy of the ELF, we plot ELF isosurfaces for a set of systems for which reference plots can be found in the literature or can be generated by other well-tested DFT programs. The EMTO code outputs the ELF as a text file in cube file format. Cube files describe three-dimensional data which have typically been evaluated on a uniform rectangular grid. One can analyze the cube file to locate the critical points, which are either minima, saddle points, or maxima. The local maxima somewhere between the atoms are the bonding attractors (assuming they are not spurious maxima caused by calculational approximations), whose location and $\eta$ value give the location and degree of covalency of the bond, respectively. We will first consider carbon in a diamond structure, as it is a prime example of strong covalent bonding. Since EMTO relies on the muffin-tin approximation, it is not the ideal method for systems with open structures, like diamond. One can, however, introduce so-called empty spheres in order to "fill the holes," which solves the problem in many cases. Figure 2 shows the ELF isosurface $\eta(\mathbf{r})=0.8$ for diamond, which has been calculated with the help of empty spheres. We can see the strong covalent bonds between carbon atoms and the EMTO figure closely reproduce the results of Ref. [4].

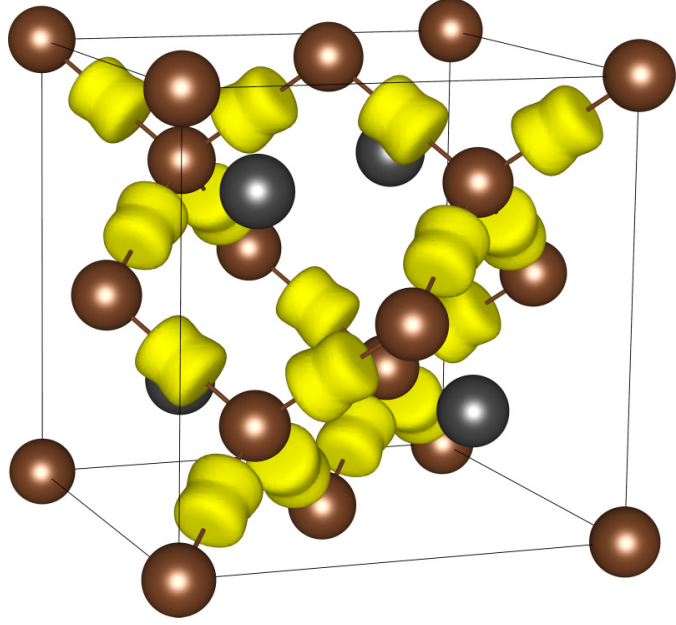

FIG. 2. ELF isosurface $\eta=0.8$ of carbon in a diamond structure. The dark gray spheres represent "empty spheres" that are added in the voids to improve the packing ratio and thus the computational accuracy.

Modeling the ELF of metallic bonds can be tricky because the ELF maxima of metallic bonds are shallower than, e.g., in the strongly covalent diamond case. It is known that the resulting ELF topology can depend quite dramatically on the computational method that is used to generate it. Fullpotential and non-full-potential methods sometimes disagree on the number and location of ELF bonding maxima [3,4]. The choice of which electrons should be included as valence electrons can also influence the ELF topology. The bulk elements that are tested include $\mathrm{Li}$ in a bcc structure and $\mathrm{Al}, \mathrm{Ca}$, and $\mathrm{Cu}$ in a fcc structure. The lattice constants of $\mathrm{Li}, \mathrm{Al}, \mathrm{Ca}$, and $\mathrm{Cu}$ were $3.49,4.05,5.56$, and $3.61 \AA$, respectively. We also calculate the binary alloy AlRu in the $B 2$ structure [43], for which the lattice constant $3.00 \AA$ was used. To showcase the CPA ELF we calculate a fcc $\mathrm{Cu}_{0.75} \mathrm{Zn}_{0.25}$ random alloy using both CPA and a supercell approach, and the results are discussed in the next section.

The isosurfaces of the elemental metals are shown in Fig. 3. Results for the same set of elements can be found in Ref. [4], where three different methods, linear muffin-tin orbital + atomic sphere approximation (LMTO-ASA), [29], Hartree-Fock (HF) [44], and Full-Potential Local-Orbital minimum-basis code (FPLO) [45], were compared. For bcc $\mathrm{Li}$ we find attractors at the octahedral voids (cell coordinates $1 / 2,1 / 2,0)$, and the ELF topology is the same as that of the FPLO method in Refs. [3,4]. For fcc Al EMTO predicts bonding attractors between the nearest-neighbor pairs, in agreement with LMTO-ASA. For fcc Ca EMTO predicts attractors only in the octahedral voids (cell coordinates $1 / 2,1 / 2,1 / 2)$, again in agreement with LMTO-ASA. The fact that in the $\mathrm{Al}$ and $\mathrm{Ca}$ cases EMTO agrees with LMTOASA and not with the full-potential code FPLO is reasonable given that EMTO is not full potential, just like LMTO-ASA. For fcc $\mathrm{Cu}$ EMTO predicts attractors in the tetrahedral voids (cell coordinates 1/4, 1/4,1/4), which agrees with both the LMTO-ASA and FPLO results.

The binary alloy AlRu is known as a good candidate for high-temperature applications, and in Fig. 4 we plot the ELF 

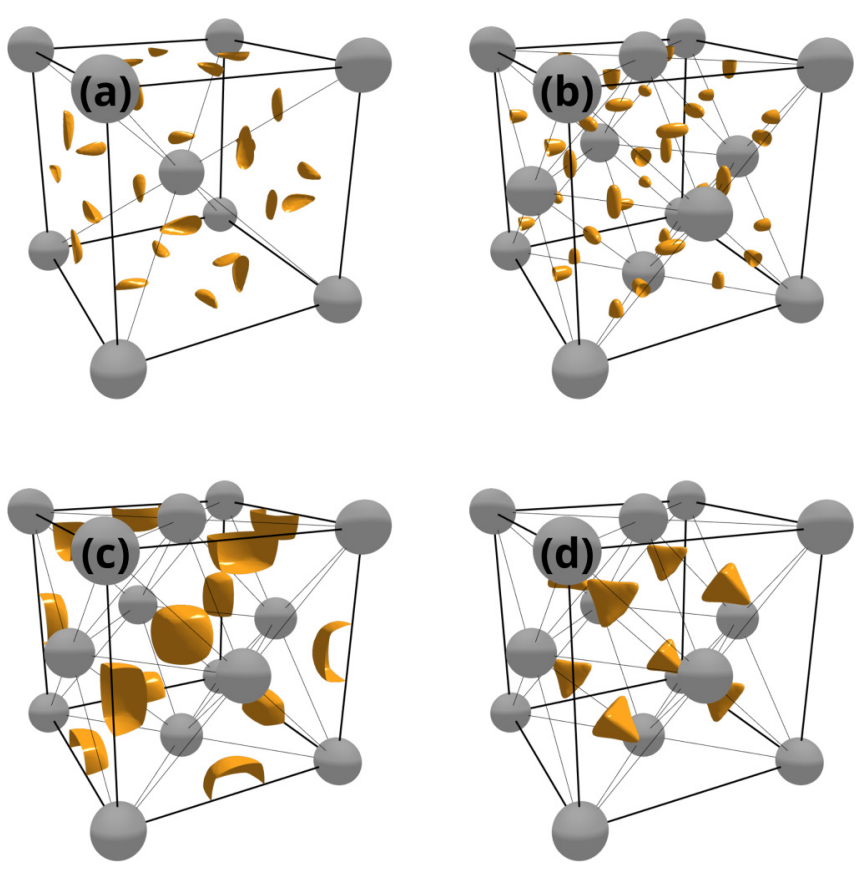

FIG. 3. ELF isosurfaces of (a) bcc Li $(\eta=0.658)$, (b) fcc Al $(\eta=0.565)$, (c) fcc Ca $(\eta=0.750)$, and (d) fcc $\mathrm{Cu}(\eta=0.270)$.

maxima using both EMTO [Fig. 4(a)] and ELK [Fig. 4(b)]. We can see that the EMTO and ELK ELF topologies are otherwise the same, with the exception of Al-Al bonding maxima (green isosurfaces, $\eta \approx 0.5$ ) that EMTO finds and ELK does not. These Al-Al maxima are the same as what can be seen in Fig. 3(b), and similar to the bulk fcc Al case, they are not predicted by full-potential methods [3]. The Ru-Ru maxima in the octahedral voids are found by both methods (red isosurfaces, $\eta \approx 0.4$ ), as are the maxima close to the $\mathrm{Ru}$ atoms (blue isosurfaces, $\eta \approx 0.8$ ). Both methods also produce the fourfold maxima close to the octahedral holes (orange isosurfaces, $\eta \approx 0.47$ ), which together with the red isosurfaces at the octahedral holes signify metallic multicenter bonding.

As discussed in Sec. II D, the interstitial region of the fcc structure requires care to be treated accurately. The center of the fcc interstitial region, where the corners of the Voronoi cells touch, is not covered by the potential spheres, and instead, the potential there is given by the constant muffin-tin zero. We have noticed that for best ELF accuracy the value of the muffin-tin zero should be checked as well. In our current case, the muffin-tin zero of fcc $\mathrm{Cu}$ had to be raised by 0.2 Ry above its default value in the self-consistent loop. The motivation for this choice will be given later. Using the default muffin-tin zero produces "unwanted" extra ELF maxima in the octahedral voids (see fcc $\mathrm{Ca}$ in Fig. 3). The reason for this is that the default muffin-tin zero does not describe the real potential accurately in the octahedral void centers in the fcc structure. Figure 5 illustrates this problem. In the octahedral void center (vertical dashed line), where the potential spheres do not overlap, the EMTO potential is assumed to be constant, and its value is fixed to the muffin-tin zero. The default muffin-tin zero produces a self-consistent potential that severely underestimates the ELK full potential in the void center (bottom panel). We have also plotted the non-self-consistent EMTO full potential calculated from the (a)

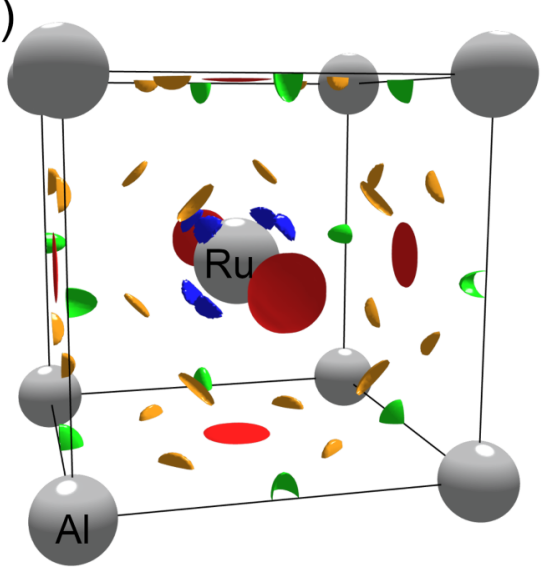

(b)

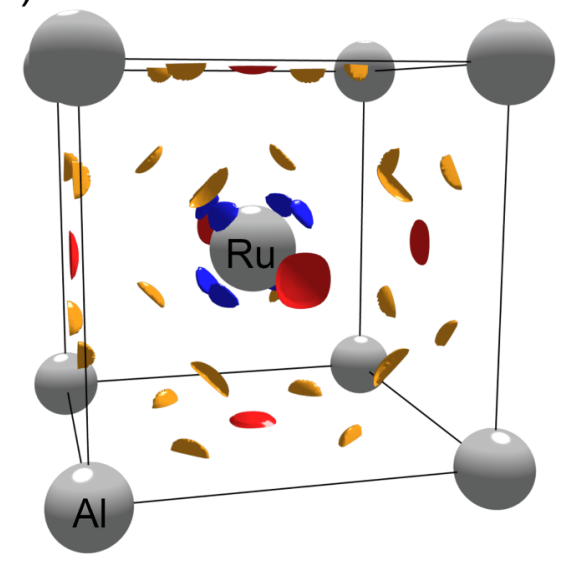

FIG. 4. The ELF bonding maxima of AlRu in the $B 2$ structure calculated with (a) EMTO and (b) elk methods.

converged density, and like the self-consistent potential, it also underestimates the ELK full potential. As a consequence, the EMTO density is overestimated in the void center, compared to a density produced by the full-potential code ELK. On the other hand, as Fig. 5 shows, the KED $\tau$ (and $\tau_{\mathrm{W}}$ ) is far less affected by the value of the muffin-tin zero due to symmetry and its dependence on the gradient operator (which vanishes at the cell boundary due to the symmetry). The overestimated density causes the denominator $\tau_{\text {unif }}$ in Eq. (2) to be too large compared to the KED-based numerator $\tau-\tau_{\mathrm{W}}$. Using the default muffin-tin zero therefore causes $\alpha(\mathbf{r})$ to have spurious minima in the octahedral void centers, which in turn means that the ELF will have maxima or attractors there. If we shift the muffin-tin zero up by $0.2 \mathrm{Ry}$, the interstitial potential is brought to better agreement with a full potential calculated with ELK (Fig. 5, bottom panel). Consequently, the agreement between EMTO and elk densities (and potentials) is dramatically improved, and the correct EMTO ELF topology is recovered.

It should be noted that the fcc Cu ELF can also be fixed by calculating it with empty spheres following the methodology of Al-Zoubi et al. [46]. As shown in Fig. 5, empty sphere calculation yields an EMTO full potential that is very similar to the ELK full potential (see the bottom panel). The empty sphere calculation can therefore be used to find the optimal 

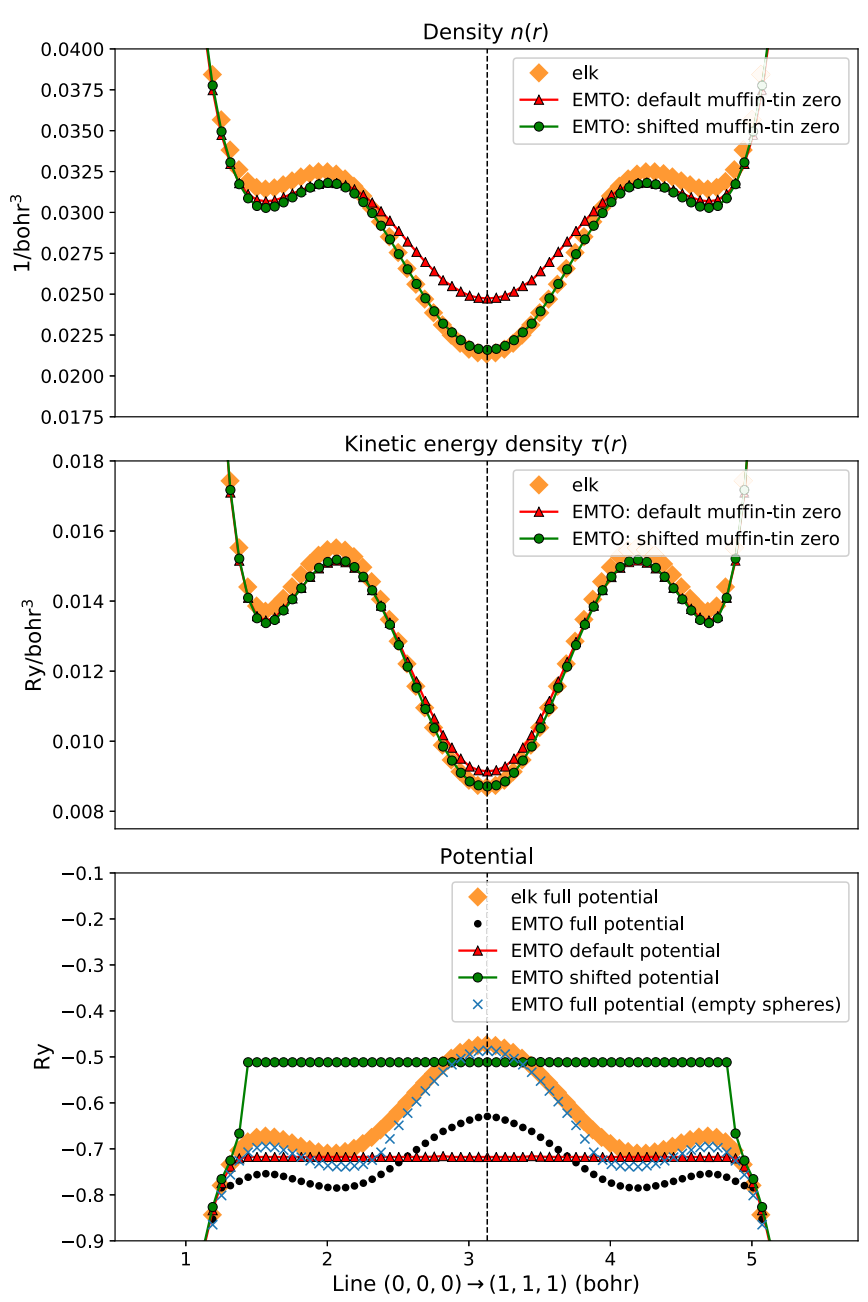

FIG. 5. fcc $\mathrm{Cu}$ line plots from $(0,0,0)$ to the $(1,1,1)$ conventional unit cell coordinate for the density, kinetic energy density, and selfconsistent potential around the octahedral void center region (dashed vertical line). Elk and non-self-consistent EMTO full potentials are also shown for comparison.

muffin-tin shift that is needed in the original nonempty sphere case. We notice, however, that introducing empty spheres in a close-packed lattice leads to additional problems (increased overlap error, semicore states, basis set convergency, etc.). Therefore, empty spheres are not a systematic way to improve results, so here we have used empty spheres to justify, based merely on EMTO calculations, why the 0.2 Ry muffin-tin zero shift needs to be made.

\section{ELF for random alloys}

It is an interesting question how well the ELF calculated with CPA compares to the ELF calculated using a supercell. Since CPA is a single-site approximation and therefore does not provide the full local environment around atomic sites the way supercells do, some ELF information can be expected to be lost. As a test case we have calculated fcc $\mathrm{Cu}_{0.75} \mathrm{Zn}_{0.25}$ with both CPA and a 32-atom special quasirandom structure (SQS) supercell [47]. The SQS supercell coordinates are taken from Ref. [48]. Both CPA and SQS calculations use the same volume. Atomic coordinates are not relaxed in the SQS calcu-
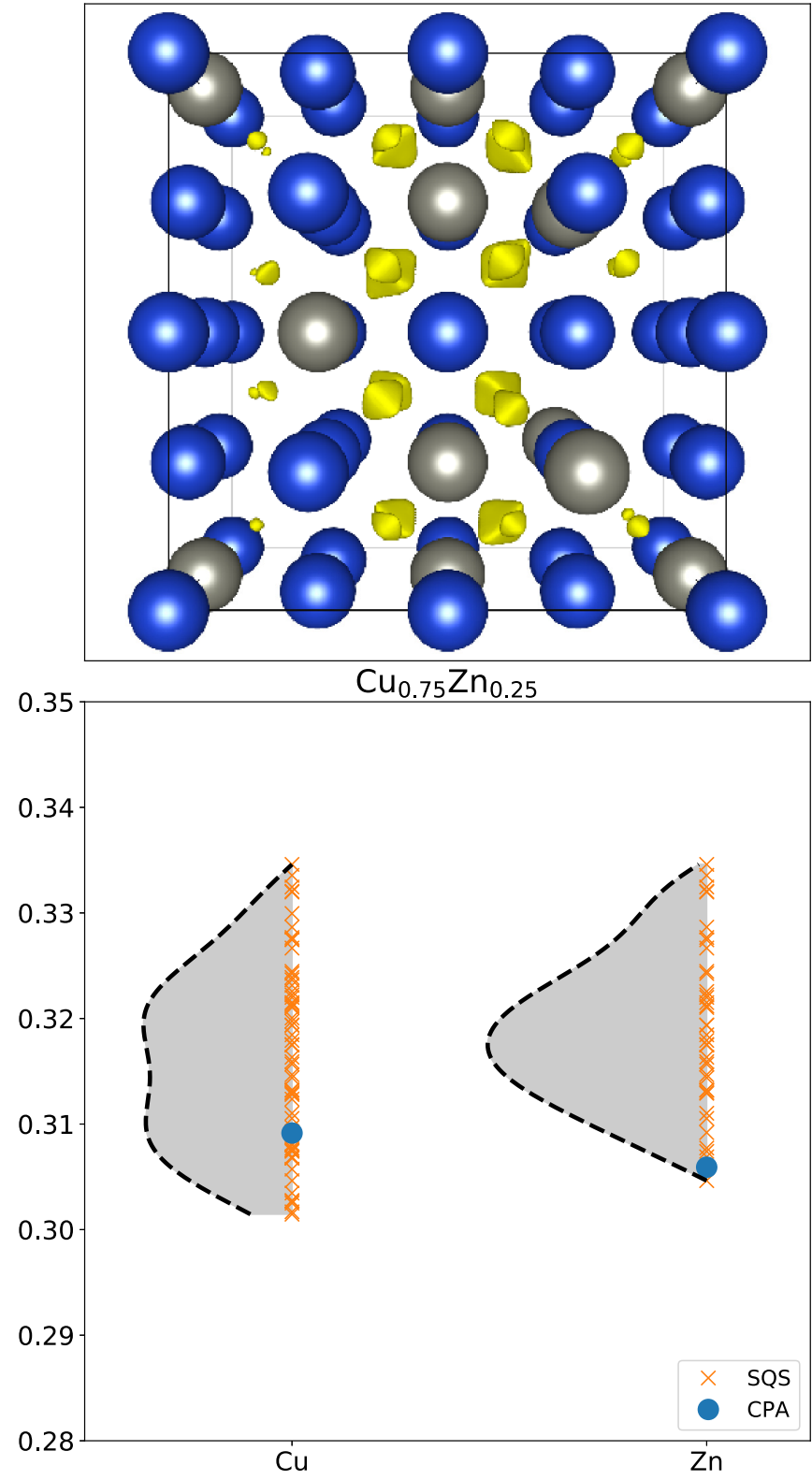

FIG. 6. Top: ELF isosurfaces of the 32-atom $\mathrm{Cu}_{0.75} \mathrm{Zn}_{0.25}$ SQS for isosurface level $\eta=0.315$. Blue spheres are $\mathrm{Cu}$ atoms, and gray spheres are Zn atoms. Bottom: Distribution of the SQS and CPA ELF bonding center maxima.

lation in order to rule out the effect of local lattice relaxations in the SQS results. As we did with bulk fcc $\mathrm{Cu}$, the muffin-tin zero is shifted up by 0.2 Ry.

The differences in CPA and supercell ELF are quantified by comparing the values of the ELF maxima in the metallic bonding centers at the tetrahedral voids. The local environment present in the supercell gives rise to a distribution of ELF bonding maxima values, whereas CPA gives only a single unique value for the maxima per CPA component due to the symmetry-retaining single-site nature of CPA. The spread of SQS ELF maxima is illustrated in the top panel of Fig. 6. The image shows ELF isosurfaces drawn for the value $\eta=0.315$. Blue spheres are $\mathrm{Cu}$ atoms, and gray spheres are $\mathrm{Zn}$ atoms. Each yellow surface in the image surrounds one of the metallic 
bonding centers. The smaller the size of the surface is, the closer the ELF maximum value of that particular bonding center is to the value 0.315. Large surface size indicates that the ELF maximum value is above 0.315 , and for empty locations the maximum value is somewhere below 0.315 . The distribution of SQS ELF maxima is collected in the bottom panel of Fig. 6, shown by orange crosses. Points are categorized to $\mathrm{Cu}$ and $\mathrm{Zn}$ maxima based on whether the bonding maxima, which are shared by four atoms, include $\mathrm{Cu}$ or $\mathrm{Zn}$ atoms. If the maxima are shared by both types of atoms, they are counted as both a $\mathrm{Cu}$ data point and a $\mathrm{Zn}$ data point. The gray area gives a kernel-density estimate (KDE) fitted to the spread of SQS ELF maxima. The CPA calculation yields just one unique value for the ELF maximum (for both $\mathrm{Cu}$ and $\mathrm{Zn}$ ), which in Fig. 6 is shown by blue symbols. It can be seen that the $\mathrm{Cu}$ CPA value agrees relatively well with the average of the SQS values, aligning with the lower peak of the KDE distribution. The lower peak corresponds to a Cu-rich environment, and the upper peak corresponds to a mixed $\mathrm{Cu}-\mathrm{Zn}$ environment. For $\mathrm{Zn}$ the agreement is not as good, but CPA still gives an estimate for the ELF maximum that is within the spread of SQS values. We are not sure what causes the discrepancy between the SQS and CPA values for $\mathrm{Zn}$. We note that the present 32-atom SQS supercell is relatively small, which might be one of the reasons behind the discrepancy. In conclusion, the CPA ELF can be seen as an approximation of the spatial average of the SQS ELF. This is, perhaps, not surprising given that CPA has been shown to agree well with average quantities extracted from SQS calculations, for example, elastic constants [49].

\section{CONCLUSIONS}

We have implemented the calculation of ELF in the EMTO DFT code. The ELF can be plotted or otherwise analyzed to study bonding characteristics or used internally by EMTO to perform calculations at the meta-GGA level. Since EMTO supports the simulation of disordered system using the CPA formalism, we have implemented the ELF in such a way that it can also accommodate CPA calculations.

EMTO predicts ELF topologies with accuracy that is somewhere between LMTO-ASA and full-potential methods (e.g., FPLO). In the bcc Li case EMTO agrees with FPLO, while LMTO-ASA produces a different topology. For the rest of the tested bulk elements EMTO agrees with LMTO-ASA. For EMTO one of the most notable approximations that affect the ELF is that EMTO assumes the potential inside the muffin-tin spheres is spherically symmetric and constant outside; that is, the currently employed EMTO code is not a full-potential method. There are currently no robust rules to tell us which method gives the "correct" ELF topology for which system. However, it is thought that full-potential methods, such as FPLO and ELK, that describe the potential without any shape approximations should generally be able to give more accurate ELF in the interstitial region far away from the WignerSeitz sphere boundary than non-full-potential methods. The true advantage of the present implementation is that it can easily be applied to chemically and magnetically random systems.

\section{ACKNOWLEDGMENTS}

The authors acknowledge the Swedish Research Council (VR), the Swedish Foundation for Strategic Research (SSF), the Swedish Foundation for International Cooperation in Research and Higher Education (STINT), and the Hungarian Scientific Research Fund (OTKA 128229). The computational resources provided by the Swedish National Infrastructure for Computing (SNIC) at Linköping are acknowledged.
[1] A. D. Becke and K. E. Edgecombe, J. Chem. Phys. 92, 5397 (1990).

[2] A. Savin, Angew. Chem., Int. Ed. Engl. 36, 1808 (1997).

[3] A. Ormeci, H. Rosner, F. R. Wagner, M. Kohout, and Y. Grin, J. Phys. Chem. A 110, 1100 (2006).

[4] Y. Grin, A. Savin, and B. Silvi, The ELF Perspective of chemical bonding, in The Chemical Bond (John Wiley \& Sons, Ltd., 2014), Chap. 10, pp. 345-382.

[5] P. Fuentealba, E. Chamorro, and J. C. Santos, Understanding and using the electron localization function, in Theoretical Aspects of Chemical Reactivity, Theoretical and Computational Chemistry, edited by A. Toro-Labbé, Vol. 19 (Elsevier, 2007), pp. 57-85.

[6] F. Fuster, A. Sevin, and B. Silvi, J. Phys. Chem. A 104, 852 (2000).

[7] Y. Zhang, Y. Zhuang, A. Hu, J. Kai, and C. Liu, Scr. Mater. 130, 96 (2017).

[8] S. Wang, T. Zhang, H. Hou, and Y. Zhao, Phys. Status Solidi B 255, 1800306 (2018).

[9] P. Hohenberg and W. Kohn, Phys. Rev. 136, B864 (1964).

[10] W. Kohn and L. J. Sham, Phys. Rev. 140, A1133 (1965).

[11] A. D. Becke and M. R. Roussel, Phys. Rev. A 39, 3761 (1989).
[12] S. K. Ghosh and R. G. Parr, Phys. Rev. A 34, 785 (1986).

[13] J. Tao, J. P. Perdew, V. N. Staroverov, and G. E. Scuseria, Phys. Rev. Lett. 91, 146401 (2003).

[14] J. P. Perdew and A. Zunger, Phys. Rev. B 23, 5048 (1981).

[15] J. P. Perdew and Y. Wang, Phys. Rev. B 45, 13244 (1992).

[16] S. H. Vosko, L. Wilk, and M. Nusair, Can. J. Phys. 58, 1200 (1980).

[17] D. C. Langreth and M. J. Mehl, Phys. Rev. B 28, 1809 (1983).

[18] S.-K. Ma and K. A. Brueckner, Phys. Rev. 165, 18 (1968).

[19] J. P. Perdew, K. Burke, and Y. Wang, Phys. Rev. B 54, 16533 (1996).

[20] J. P. Perdew and W. Yue, Phys. Rev. B 33, 8800 (1986).

[21] J. P. Perdew, Phys. Rev. Lett. 55, 1665 (1985).

[22] J. P. Perdew, J. A. Chevary, S. H. Vosko, K. A. Jackson, M. R. Pederson, D. J. Singh, and C. Fiolhais, Phys. Rev. B 46, 6671 (1992).

[23] Y. Zhao and D. G. Truhlar, J. Chem. Phys. 128, 184109 (2008).

[24] F. R. Wagner, http://www2.cpfs.mpg.de/ELF/index.php? content=06interpr.txt.

[25] J. Sun, A. Ruzsinszky, and J. P. J. Perdew, Phys. Rev. Lett. 115, 036402 (2015).

[26] J. Tao and Y. Mo, Phys. Rev. Lett. 117, 073001 (2016). 
[27] J. P. Perdew and L. A. Constantin, Phys. Rev. B 75, 155109 (2007).

[28] R. Tank and C. Arcangeli, Phys. Status Solidi B 217, 89 (2000).

[29] O. K. Andersen, Phys. Rev. B 12, 3060 (1975).

[30] H. L. Skriver, The LMTO Method, Springer Series in Solid-State Sciences Vol. 41 (Springer, Berlin, 1984).

[31] O. K. Andersen and O. Jepsen, Phys. Rev. Lett. 53, 2571 (1984).

[32] O. K. Andersen, O. Jepsen, and G. Krier, in Lectures on Methods of Electronic Structure Calculations, edited by V. Kumar, O. K. Andersen, and A. Mookerjee (World Scientific, Singapore, 1995), pp. 63-124.

[33] L. Vitos, Phys. Rev. B 64, 014107 (2001).

[34] L. Vitos, Computational Quantum Mechanics for Materials Engineers, Engineering Materials and Processes (Springer, London, 2007).

[35] A. Östlin, L. Vitos, and L. Chioncel, Phys. Rev. B 96, 125156 (2017).

[36] E. N. Economou, Green's Functions in Quantum Physics, Springer Series in Solid-State Sciences Vol. 7 (Springer, Berlin, 2006).
[37] I. A. Abrikosov and B. Johansson, Phys. Rev. B 57, 14164 (1998).

[38] B. L. Gyorffy, Phys. Rev. B 5, 2382 (1972).

[39] P. Soven, Phys. Rev. 156, 809 (1967).

[40] A. D. Becke, J. Chem. Phys. 88, 2547 (1988).

[41] H. Levämäki, M. Kuisma, and K. Kokko, J. Chem. Phys. 150, 054101 (2019).

[42] K. Dewhurst, http://elk.sourceforge.net/.

[43] I. Horner, N. Hall, L. Cornish, M. Witcomb, M. Cortie, and T. Boniface, J. Alloys Compd. 264, 173 (1998).

[44] V. Fock, Z. Phys. 61, 126 (1930).

[45] K. Koepernik and H. Eschrig, Phys. Rev. B 59, 1743 (1999).

[46] N. I. Al-Zoubi, M. P. J. Punkkinen, B. Johansson, and L. Vitos, Phys. Rev. B 81, 045122 (2010)

[47] A. Zunger, S.-H. Wei, L. G. Ferreira, and J. E. Bernard, Phys. Rev. Lett. 65, 353 (1990).

[48] J. von Pezold, A. Dick, M. Friák, and J. Neugebauer, Phys. Rev. B 81, 094203 (2010).

[49] S. Huang, F. Tian, and L. Vitos, J. Mater. Res. 33, 2938 (2018). 